\title{
PSEUDO-RIEMANNIAN METRICS AND HIRZEBRUCH SIGNATURE
}

\author{
PETER R. LAW
}

(Communicated by Jonathan M. Rosenberg)

\begin{abstract}
On compact, orientable, $4 k$-dimensional manifolds, nonvanishing Hirzebruch signature is shown to be an obstruction to the existence of certain kinds of pseudo-Riemannian metrics.
\end{abstract}

Consider a compact, orientable, $4 k$-dimensional manifold $M$. The bilinear mapping

$$
Q(\alpha, \beta):=\int_{M} \alpha \wedge \beta
$$

on the de Rham cohomology group $H^{2 k}(M, \mathbf{R})$ is a symmetric, nondegenerate bilinear form. Suppose it is of signature type $(p, q)$. The number $\tau:=p-q$ is called the Hirzebruch signature and is a topological invariant of $M$. (Consult, for example, [2, p. 224] for properties of $\tau$, and translate into differential forms via the de Rham isomorphism of cohomology algebras.) With respect to a Riemannian metric on $M$, the Hodge theory of harmonic differential forms allows one to replace the space $H^{2 k}(M, \mathbf{R})$ by the space $H^{2 k}$ of harmonic $2 k$-forms on $M$. Since the Hodge star operator $*$ commutes with the Hodge Laplacian $\Delta$, then $*$ induces a linear isomorphism of $H^{2 k}$.

A pseudo-Riemannian metric $g$ on $M$ also defines a Hodge star operator and a Hodge Laplacian in an analogous fashion; but the Hodge theory of the Riemannian case need not be valid with respect to these operators. For the remainder of this article, all metric-dependent quantities are with respect to $g$. If $E^{p}$ denotes the space of global $p$-forms on $M$, let $B^{p}, D^{p}, Z^{p}$, and $C^{p}$ denote the subspaces of $d$-exact, $\delta$-exact, $d$-closed, and $\delta$-closed forms respectively. Avez [1, pp. 168-175] calls $(M, g)$ "weakly de Rham" if $A^{p}:=$ $B^{p}+D^{p}+\left(Z^{p} \cap C^{p}\right)$ is dense (with respect to the weak topology defined by the natural inner product on $E^{p}$ ) in $E^{p}$. Avez shows that this condition entails that $H^{p}=\left(Z^{p} \cap C^{p}\right)$, whence every harmonic form is $d$-closed, and that if a cohomology class contains a harmonic representative it contains a unique such. If $A^{p}=E^{p}$, Avez calls $(M, g)$ "strongly de Rham" and demonstrates [1, $\mathrm{p}$. 181], at least in two dimensions, that the former notion is weaker than the latter. The argument in the opening paragraph is applicable to $(M, g)$ if $(M, g)$ is strongly de Rham. More generally, it is applicable if $(M, g)$ is only weakly de Rham and each cohomology class contains a harmonic representative (that

Received by the editors September 24, 1990.

1980 Mathematics Subject Classification (1985 Revision). Primary 53C50, 58A12. 
this condition is weaker than $(M, g)$ being strongly de Rham is again shown by Avez's two-dimensional examples). Call such an $(M, g)$ "weakly de Rham with harmonic cohomology."

Theorem. If $M$ is a compact, orientable, $4 k$-dimensional manifold $M$ carrying a pseudo-Riemannian metric $g$ of signature $(r, s), s$ odd (equivalently $r$ odd), such that $(M, g)$ is weakly de Rham with harmonic cohomology then the Hirzebruch signature of $M$ vanishes.

Proof. For any two forms of equal degree, $\alpha \wedge * \beta=\beta \wedge * \alpha$, so for $\alpha$ and $\beta$ in $H^{2 k}$ one has $Q(* \alpha, * \beta)=\int_{M} * \alpha \wedge * \beta=\int_{M} \beta \wedge * * \alpha=(-1)^{2 k(2 k)+s} \int_{M} \beta \wedge \alpha=$ $(-1)^{s} \int_{M} \alpha \wedge \beta=(-1)^{s} Q(\alpha, \beta)$, i.e. on $H^{2 k}, Q(* \alpha, * \beta)=(-1)^{s} Q(\alpha, \beta)$. Thus, for $s$ odd, $*$ is an anti-isometry of $\left(H^{2 k}, Q\right)$, which must therefore be neutral (cf. [3, p. 164]), i.e. $\tau=0$.

Except in two dimensions, Avez [1] gave no results concerning the existence or impossibility of weakly or strongly de Rham pseudo-Riemannian metrics.

Example. The complex projective spaces $C P^{2 k}$ do not admit any pseudo-Riemannian metrics of the type stated in the theorem.

Proof. $\tau\left(\mathbf{C P}^{2 k}\right)=1[2$, p. 225].

As an indication of the limitations of the theorem presented above, suppose $M$ admits a Riemannian metric of constant curvature. Then the Pontryagin classes $p_{1}, \ldots, p_{k}$ all vanish (cf. $[4$, p. 516]). By the Hirzebruch signature theorem [2, p. 224], it follows that $\tau$ vanishes. Thus, the theorem provides no information in such a case.

Of special interest is dimension four, in which case the metric must be Lorentzian for the theorem to be relevant and so the Euler characteristic $\chi$ must vanish. Examples of four-dimensional, compact, orientable manifolds with $\chi$ zero but $\tau$ nonzero appear to require a little effort to produce. Let $S$ be a compact, orientable, two-dimensional manifold of genus two so that $\chi(S)=-2$. Let $X_{n}$ denote the connected sum of $n$ copies of $\mathbf{C P}^{2}$ and $Y_{m}$ the connected sum of $m$ copies of $\mathbf{C P}^{1} \times S$. Using the formula

$$
\chi(M \sharp N)=\chi(M)+\chi(N)-2 \text {, }
$$

valid for connected, orientable manifolds $M$ and $N$, properties of $\tau$, and the fact that $\tau\left(\mathbf{C P}^{2 k}\right)=1$, one easily determines:

$$
\begin{array}{cc}
\chi\left(X_{n}\right)=n+2, & \tau\left(X_{n}\right)=n, \\
\chi\left(Y_{m}\right)=2-6 m, & \tau\left(Y_{m}\right)=0 .
\end{array}
$$

Hence, one finds

$$
\chi\left(X_{n} \sharp Y_{m}\right)=n-6 m+2, \quad \tau\left(X_{n} \sharp Y_{m}\right)=n .
$$

Reversing the orientation of $\mathbf{C P}^{2}$ reverses the sign of $\tau$ in these formulae.

Example. $M_{m}:=X_{n} \sharp Y_{m}$, with $n=6 m-2$ and $m$ any positive integer, are compact, orientable, four-dimensional manifolds admitting Lorentz metrics that cannot be weakly de Rham with harmonic cohomology.

An example of a compact, orientable, four-dimensional Lorentz manifold that is weakly de Rham with harmonic cohomology can be produced by extending Avez's two-dimensional example [1, pp. 175-177]. 
Example. Let $M$ be $\mathbf{S}^{1} \times \mathbf{S}^{1} \times \mathbf{S}^{1} \times \mathbf{S}^{1}$ equipped with the "pseudo-Euclidean" metric

$$
g:=\left(\sigma^{1}\right)^{2}-\mu^{2}\left[\left(\sigma^{2}\right)^{2}+\left(\sigma^{3}\right)^{2}+\left(\sigma^{4}\right)^{2}\right],
$$

where $\sigma^{i}$ is the pullback of the standard global 1 -form on $\mathbf{S}^{1}$ by the projection onto the $i$ th factor and $\mu^{2}$ is an irrational constant. $(M, g)$ is weakly de Rham with harmonic cohomology.

Proof. The 1-forms $\sigma^{1}, \ldots, \sigma^{4}$ provide a global frame for the cotangent bundle $T^{*}$ of $M$ (indeed, a pseudo-orthonormal frame when suitably normalized) and global frames for each $\Lambda^{k}\left(T^{*}\right)$ may be formed from wedge products of the $\sigma^{i}$. Moreover, by the Künneth formula, these various forms may be taken as representatives of the distinct cohomology classes of $M$. The action of the Hodge star operator with respect to $g$ on these forms is easily determined and one finds that they lie in $Z^{p} \cap C^{p}$ for the appropriate $p$, and thus are harmonic.

Let $L^{p}:=\Delta\left(E^{p}\right)$ and denote by $R^{p}$ the subspace of $E^{p}$ whose elements have constant components when expressed in terms of the global frame derived from the $\sigma^{i}$. The Fourier series argument of Avez [1, p. 176] extends to the present example with the same conclusion that $L^{0}+R^{0}$ is dense in $E^{0}$. It is in this argument that the irrationality of $\mu^{2}$ is vital. Of course, $L^{0}$ is contained in $B^{0}+D^{0}$ and $R^{0}$ in $Z^{0} \cap C^{0}$. Now $E^{1} \simeq\left(E^{0}\right)^{4}$, via the global frame mentioned above. Writing $\Phi=\sum_{i=1}^{4} f_{i} \sigma^{i}$ for $\Phi$ in $E^{1}$ then, because $\sigma^{i}=d \theta^{i}$ for obvious local coordinates $\left\{\theta^{1}, \ldots, \theta^{4}\right\}$ in a neighborhood of any point and because the metric is pseudo-Euclidean, the Weitzenböck formula yields $\Delta \Phi=\sum_{i=1}^{4}\left(\Delta f_{i}\right) \sigma^{i}$. Thus, $L^{1}$ may be identified with $\left(L^{0}\right)^{4}$ and $R^{1}$ with $\left(R^{0}\right)^{4}$ under the linear homeomorphism $E^{1} \simeq\left(E^{0}\right)^{4}$. Thus the density of $L^{0}+R^{0}$ in $E^{0}$ entails the density of $L^{1}+R^{1}$ in $E^{1}$, and consequently the density of $B^{1}+D^{1}+\left(Z^{1} \cap C^{1}\right)$ in $E^{1}$. The same argument may be applied to $E^{p}$ for $p=2,3,4$. Note also that the density result for a given $p$ follows from the density result for $n-p$ by application of $*$.

Finally, note that the proof of the theorem requires only that one can replace $H^{2 k}(M, \mathbf{R})$ by $H^{2 k}$. This is not just a condition on forms of degree $2 k$, however. If $\alpha$ is a harmonic $(2 k-1)$-form that is $\delta$-closed but not $d$-closed, then $\Delta(d \alpha)=d \delta d \alpha=d(\Delta \alpha)=0$, whence harmonic representatives of elements of $H^{2 k}(M, \mathbf{R})$ will not be unique.

\section{ACKNOWLEDGMENT}

I am grateful to Nick Buchdahl for a stimulating conversation in connection with the penultimate example.

\section{BIBLIOGRAPHY}

1. A. Avez, Essais de géométrie Riemannienne hyperbolique globale-applications a la relativité générale, Ann. Inst. Fourier (Grenoble) 13 (1963), 105-190.

2. J. W. Milnor and J. D. Stasheff, Characteristic classes, Princeton Univ. Press, Princeton, NJ, 1974. 
3. I. R. Porteous, Tonological geometry, 2nd ed., Cambridge Univ. Press, Cambridge, 1981.

4. M. Spivak, A comprehensive introduction to differential geometry, vol. V, 2nd ed., Publish or Perish, Berkeley, 1979.

Department of Mathematics, Tufts University, Medford, Massachusetts 02155

Current address: Mathematical Reviews, 416 Fourth Street, P.O. Box 8604, Ann Arbor, Michigan 48107-8604 